\title{
Electric Transport Properties of Alkali Polymethacrylates in Alkali Bromide Solutions
}

\author{
W. P. J. T. VAN DER DRIFT AND J. TH. G. OVERBEEK \\ Van't Hoff Laboratory for Physical and Colloid Chemistry, State University of Utrecht, \\ Padualaan 8, Utrecht, The Netherlands
}

Received January 23, 1979; accepted February 6, 1979

\begin{abstract}
Electric mobilities of polyions, bromide ions, and alkali ions have been determined in solutions of $\mathrm{Li}, \mathrm{Na}$, and $\mathrm{K}$ salts of polymethacrylic acid (PMA) in aqueous solutions of the corresponding bromide of concentrations varying from 0.001 to $0.1 \mathrm{M}$. The Hittorf method was used for the determination of the mobilities of the PMA ion and of the $\mathrm{Br}^{-}$ion. The mobilities of the alkali ions followed from these two mobilities and the conductivity. A few moving boundary experiments have been carried out with tetramethylammonium PMA. The mobility of PMA was independent of the PMA concentration and of the kinds of cations and changed only slowly with the degree of neutralization. The bromide mobility decreased slowly with increasing PMA concentration. The interpretation was based upon a separation of the contributions from the polyelectrolyte salt and those of the supporting electrolytes. The mobilities of the counterions that neutralized the polyions were very low and in several cases even negative. Electrophoretic retardations varied from about 20 to $70 \Omega^{-1} \mathrm{~cm}^{2} \mathrm{eq}^{-1}$. By using the relaxation correction as found from conductivity data we could interpret the mobility of the PMA ion as that of a cylinder of $0.35-\mathrm{nm}$ radius surrounded by a solvation layer of $0.2-$ to 0.3 -nm thickness. For bromide concentrations up to $0.03 \mathrm{M}$ the agreement was excellent; at a $0.1 \mathrm{M}$ bromide concentration binding of about $25 \%$ of the counterions to the polyions had to be assumed to reconcile the model calculations with the experiments. Intrinsic viscosities of $\mathrm{NaPMA}$ in $\mathrm{NaBr}$ solutions have been used to obtain an estimate of the overall size of the polyelectrolyte coil and to justify the cylinder model.
\end{abstract}

\section{INTRODUCTION}

The electric properties of polyelectrolytes are strongly influenced by the concentration of low-molecular-weight electrolytes in the solution. To obtain a complete picture of the transport of electricity in polyelectrolyte solutions, the values of the conductivity and the separate mobilities of polyions and small ions are required. In this paper we give the results of measurements at $25^{\circ} \mathrm{C}$ of the mobilities in a three-ion system (polymethacrylate (PMA), bromide, and alkali ions), obtained with the Hittorf transference method.

We have chosen PMA as the polyion, since its charge can be varied by varying the degree of neutralization, $\alpha$, and its mobility is independent of the kinds of alkali counterions (1) and independent of the molecular weight $(2,3)$. The bromide ion was chosen as the co-ion because its concentration can be determined very accurately by potentiometric titration. $\mathrm{Li}, \mathrm{Na}, \mathrm{K}$, and, in a few cases, tetramethylammonium (TMA) have been chosen as the counterions. The intrinsic viscosity has been determined for auxiliary information (see Appendix).

Properties of polyelectrolytes have been interpreted by considering the polyelectrolyte coil either as a homogeneous porous sphere, or as a randomly kinked cylinder. In a previous paper (4) we have indicated that the overall size of the coil, as, e.g., derived from viscosity data, is so large and the interaction between distant parts of the same chain so small, that the model of the porous sphere has to be rejected in favor of the cylinder model. 
When we made our measurements no complete theory of the electric mobility of cylindrical particles was available. Therefore we base our interpretation on a theory of electrophoresis of cylinders, neglecting the relaxation effect, and apply a semiempirical correction for relaxation. This correction, as first used by Möller et al. (5), is based upon the idea that the structure of the double layer around the polyion is independent of the type of monovalent counterion, that, therefore, the electrophoretic retardation, $\lambda^{\prime}$, has the same value for all counterions, and that the relaxation effect can be effectively taken into account by assuming that both the polyion and the counterions that compensate its charge move in a field, $X-\Delta X$, where $X$ is the applied field strength and $\Delta X$ the average relaxation field strength. This assumption satisfies the requirement formulated by Onsager (6) that the relaxation forces on all the ions must add up to zero.

The mobilities of the ions of the supporting electrolyte are assumed to be independent of the concentration of added alkali PMA. Then the conductivities of XPMA and $\mathrm{XBr}(\mathrm{X}=\mathrm{Li}, \mathrm{Na}$, or $\mathrm{K})$ are additive and the equivalent conductivity $\lambda_{i}^{*}$ of the cations compensating the charge of the polyion can be found by a simple subtraction. On this basis the equivalent conductivities are given by

$$
\lambda_{i}^{*}=\left(\lambda_{i}^{\infty}-\lambda^{\prime}\right)(1-\Delta X / X)
$$

and

$$
\lambda_{\text {PMA }}^{*}=\left(\lambda_{\text {PMA }}^{\text {n.r. }}\right)(1-\Delta X / X) .
$$

In Eq. [1] $\lambda_{i}^{\infty}$ is the equivalent conductivity of the counterion, $i$, at infinite dilution. In Eq. [2] $\lambda_{P M A}^{n} \cdot$ is $_{\text {. }}$ is the equivalent conductivity of the polyion in the relevant bromide concentration calculated while neglecting the relaxation effect. Stigter (7) recently worked out a theory for the electrophoresis of randomly coiled cylinders and applied it to our data. We come back to the differences in the two interpretations later in this paper.

\section{EXPERIMENTAL METHODS}

\section{2a. Materials}

The purification of nitrogen, the drying of the alkali bromides, and the preparation of PMA, conductivity water, and carbonatefree alkali hydroxides have been described elsewhere (8). PMA was prepared by free radical polymerization with $\mathrm{H}_{2} \mathrm{O}_{2}$ as the initiator. After fractionation the molecular weight was 270,000 . The titration procedure was calibrated with very pure $\mathrm{KBr}$ (Koch and Light, 99.999\%). $\mathrm{AgNO}_{3}$ (U.C.B., pour analyse), $\mathrm{KNO}_{3}$ (B.D.H., AnalaR), $\mathrm{NaNO}_{3}$ (B.D.H., AnalaR), acetic acid (Merck, pro analysi), and sodium acetate (Merck, pro analysi) were used without further purification.

\section{2b. Bromide Titration}

In order to obtain the required accuracy all titrations with $\mathrm{AgNO}_{3}$ were carried out on a weight basis. An aliquot of the unknown bromide solution was diluted to about $50 \mathrm{ml}$ of $0.002 \mathrm{M}$ final $\mathrm{Br}^{-}$concentration. The final solution was made $1.4 M$ in $\mathrm{KNO}_{3}, 0.2 M$ in $\mathrm{NaNO}_{3}$, and $0.03 M$ in $\mathrm{H}-\mathrm{Na}$ acetate of $\mathrm{pH}=4.5$ (9).

During the titration the potential difference was measured between an $\mathrm{Ag} / \mathrm{AgBr}$ electrode (10) and a saturated calomel electrode connected to the solution via a van Laar-type $(11,12) \quad 1.75 \mathrm{M} \mathrm{KNO}_{3}-$ $0.25 \mathrm{M} \mathrm{NaNO} \mathrm{Na}_{3}$ salt bridge. The endpoint was taken as $192.9 \mathrm{mV}$. At $150 \mathrm{mV}$ the solution was allowed to stand and coagulate for $20 \mathrm{~min}$. The results showed a standard deviation of $0.003 \%$. The small influence of PMA on the amount of $\mathrm{AgNO}_{3}$ needed to reach the endpoint (about $0.03 \%$ at equal (mono)molar concentrations of PMA and $\mathrm{Br}^{-}$) was left out of consideration, since only solutions of nearly the same PMA content were compared.

\section{2c. PMA Determination by Refractometry}

The concentrations of the polyelectrolyte XPMA $(X=\mathrm{Li}, \mathrm{Na}$, or $\mathrm{K})$ have been 
TABLE I

$d n / d c$ at Different Degrees of Neutralization, $\alpha$, in $\mathrm{Na}-\mathrm{D}$ Light at $20.0^{\circ} \mathrm{C}$

\begin{tabular}{lcccc}
\hline & \multicolumn{5}{c}{$\alpha$} \\
\cline { 2 - 5 } & 0 & 0.3 & 0.5 & 0.7 \\
\hline$d n / d c^{a}$ & 0.160 & 0.200 & 0.226 & 0.251
\end{tabular}

${ }^{a}$ In $\mathrm{ml} / \mathrm{g}$ of polymethacrylic acid.

determined by interferometry (13), using a Haber-Löwe interferometer Aus Jena, Jena, DDR. The contributions of $\mathrm{XBr}$ and XPMA to the refractive index were nearly additive. Refractive index increments depended on the degree of neutralization, $\alpha$, of the polymethacrylic acid, but only to a negligible extent on the counterion. Table I gives the values of $d n / d c$ as used. The more dilute solutions were analyzed in a thermostated $( \pm 0.002 \mathrm{~K}) 50 \mathrm{~cm}$ cell where an accuracy of about $8 \times 10^{-8}$ in $\Delta n$ was reached. For the more concentrated solutions an $8 \mathrm{~cm}$ cell was used.

\section{2d. Hittorf Method}

The Pyrex Hittorf cell is shown in Fig. 1. The stopcocks B and D are hollow and the grip could be removed to allow circulation of water of the constant-temperature $\left(25^{\circ} \mathrm{C}\right.$ $\pm 0.005 \mathrm{~K}$ ) bath, thus preventing local heating during current passage. Platinum wires $(\phi=1 \mathrm{~mm}$, total length $=12 \mathrm{~cm})$, wound in spiral form, were used as the electrodes. The platinum was fused into soft glass via a transition glass. The electrodes were electrolytically silvered in a solution of $10 \mathrm{~g} \mathrm{KAg}(\mathrm{CN})_{2}$ per liter. About $25 \%$ of the silver of the cathode was electrolytically converted into $\mathrm{AgBr}$ in a solution containing $0.1 \mathrm{MBr}$ and $0.04 \mathrm{M}$ $\mathrm{HBr}$. The silver anode was surrounded by a solution containing $20 \%$ sucrose and 0.1 or $1 M \mathrm{NaBr}$ (5). This prevented undesirable reactions of the silver ions coming from the anode with the PMA ions during current passage. The cell was filled from MacInnes flasks (14) with solutions freed of oxygen and of carbon dioxide (15). A current stabilizer kept the current constant within $0.02 \%$ at values varying from $0.5 \mathrm{~mA}$ for $0.001 \mathrm{M}$ $\mathrm{XBr}$ to $6 \mathrm{~mA}$ for $0.1 \mathrm{MXBr}$. Current was allowed to flow for $1.5 \mathrm{hr}$. The current was calculated from the voltage drop at standard resistors at the cathode and anode sides of the cell. Current leakage, manifesting itself as a difference in the current at either side of the cell, was below $0.05 \%$. Current leakage at stopcocks $\mathrm{A}$ and $\mathrm{E}$ was prevented by wrapping them in small polyethylene bags.

After the passage of the current stopcocks $\mathrm{B}$ and $\mathrm{D}$ were closed. The middle compartment was emptied by means of a pipet. The solution of the cathode compartment was drawn off through stopcock E. The compartment was rinsed three times with, in total, about $20 \mathrm{~g}$ of the original solutions. The procedure was carried out under nitrogen, since admission of air had a measurable effect on the refractive index. The contents of the cathode compartment together with the washings were weighed.

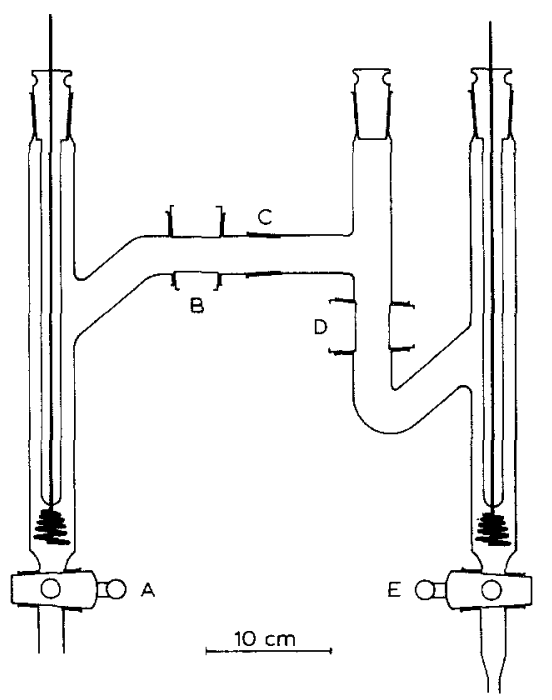

Fig. 1. Hittorf cell. Anode (A) at the left, cathode (E) at the right. The small stopcocks $A$ and $E$ and the diameter of the tube $(\phi=2 \mathrm{~cm})$ are not to scale. They are enlarged in the figure. 
Aliquots of the thoroughly homogenized solution were analyzed as described in Sections $2 \mathrm{~b}$ and $2 \mathrm{c}$. The change in bromide concentration in the middle compartment was always below $0.03 \%$. The anode compartment was not analyzed. Experiments without current passage showed that the manipulation error was below $0.01 \%$ for $\mathrm{Br}^{-}$and within the analytic error for PMA.

The equivalent conductivity, $\lambda_{\text {PMA }}^{*}$, of the partially neutralized polymethacrylate ion was found from

$$
\lambda_{\mathrm{PMA}}^{*}=\frac{\Delta m_{\mathrm{PMA}} \cdot \kappa \cdot F}{g_{\mathrm{PMA}} \cdot i \cdot t},
$$

where $\Delta m_{\mathrm{PMA}}$ is the decrease of the mass of PMA in the cathode compartment, $\kappa$ the conductivity, $F$ the Faraday constant, $g_{\text {PMA }}$ the original PMA concentration in mass/ volume, $i$ the current, and $t$ the time. The equivalent conductivity, $\lambda_{\mathrm{Br}}$, was found from

$$
\lambda_{\mathrm{Br}}=\frac{\left(m_{\mathrm{Br}}-\delta m_{\mathrm{Br}}\right) \cdot \kappa \cdot F}{g_{\mathrm{Br}} \cdot i \cdot t},
$$

where $m_{\mathrm{Br}}$ is the calculated mass of bromide ion generated at the cathode, $\delta m_{\mathrm{Br}}$ is the increase of the mass of bromide in the cathode compartment, and $g_{\mathrm{Br}}$ is the original bromide concentration in mass/volume.

The performance of the procedure was checked with four experiments with $0.01 \mathrm{M}$ $\mathrm{KBr}$ without PMA. The equivalent conductivities, $\lambda_{\mathrm{Br}}$, were found to be 73.82 , 73.55, 73.57, and 73.77 $\Omega^{-1} \mathrm{~cm}^{2} \mathrm{~mole}^{-1}$. The value obtained with the moving boundary method is $73.97 \Omega^{-1} \mathrm{~cm}^{2}$ mole $^{-1}$

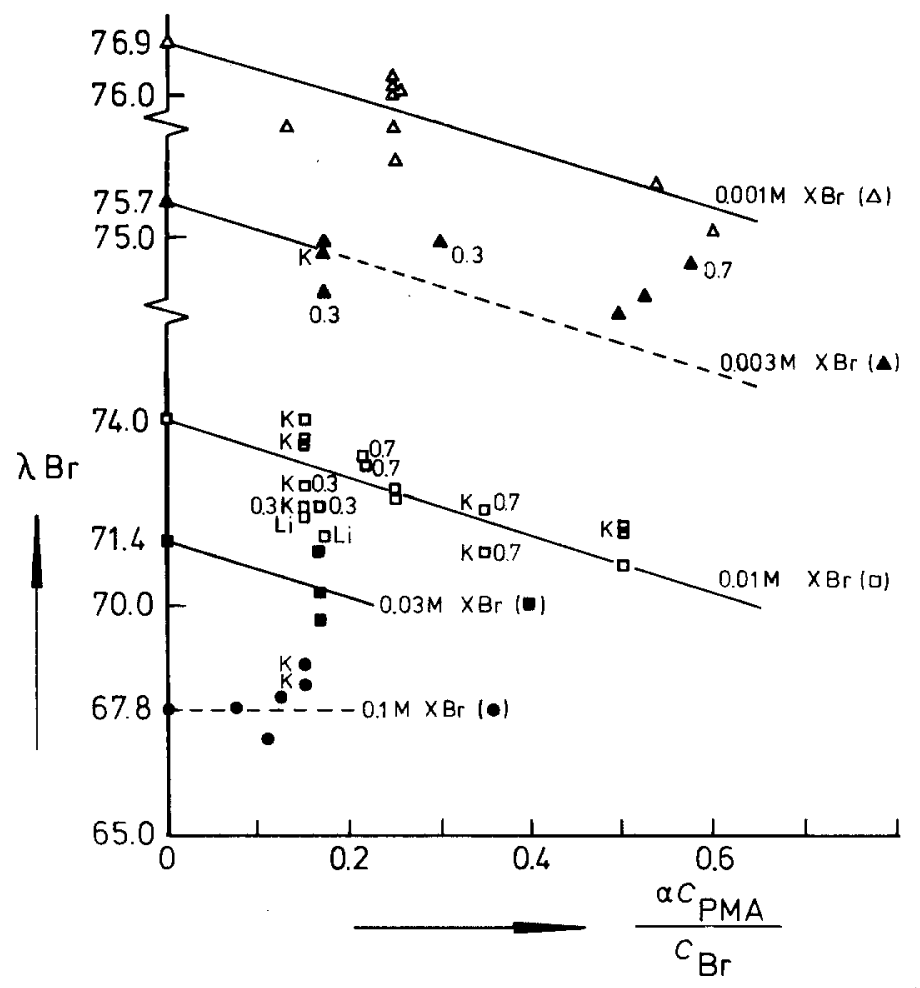

FIG. 2. Influence of the PMA concentration on the equivalent conductivity of the bromide ions at $\alpha=0.3, \alpha=0.5$, and $\alpha=0.7 . \lambda_{\mathrm{Br}}$, expressed in $\Omega^{-1} \mathrm{~cm}^{2}$ mole ${ }^{-1}$, is plotted against $\alpha c_{\mathrm{PMA}} / c_{\mathrm{Br}}$ for five concentrations of bromide. Unmarked points refer to $\mathrm{Na}$ counterions and $\alpha=0.5$. Experiments with other counterions and/or other values of $\alpha$ are indicated with the symbols $\mathrm{Li}$, $\mathrm{K}, 0.3$, and 0.7 . 
(16). The fact that the $\lambda_{\mathrm{Br}}$ values are slightly low must probably be attributed to liberation of occluded $\mathrm{Br}^{-}$from the $\mathrm{Ag} / \mathrm{AgBr}$ cathode (15). More experimental details can be found in van der Drift (17).

\section{RESULTS}

$$
\text { 3a. } \lambda_{B r}
$$

The equivalent conductivity of the bromide ions depends slightly, but notably, on the concentration of PMA. The equivalent conductivity decreases with increase of the PMA concentration, and as shown in Fig. 2 can be represented (except for $0.1 M \mathrm{XBr}$ ) within the limited accuracy of the experiments by the following empirical equation:

$$
\begin{aligned}
\lambda_{\mathrm{Br}}\left(c_{\mathrm{Br}}, c_{\mathrm{PMA}}\right) & =\lambda_{\mathrm{Br}}\left(c_{\mathrm{Br}}, c_{\mathrm{PMA}}=0\right) \\
- & \frac{6 \alpha c_{\mathrm{PMA}}}{c_{\mathrm{Br}}} \Omega^{-1} \mathrm{~cm}^{2} \mathrm{~mole}^{-1},
\end{aligned}
$$

where $c_{\mathrm{Br}}$ and $c_{\mathrm{PMA}}$ are expressed in moles and monomoles, respectively, per unit volume. The experiments with $\mathrm{Li}$ or $\mathrm{K}$ as counterions gave the same values for the equivalent conductivities of the bromide and PMA ions as the corresponding measurements with $\mathrm{Na}$.

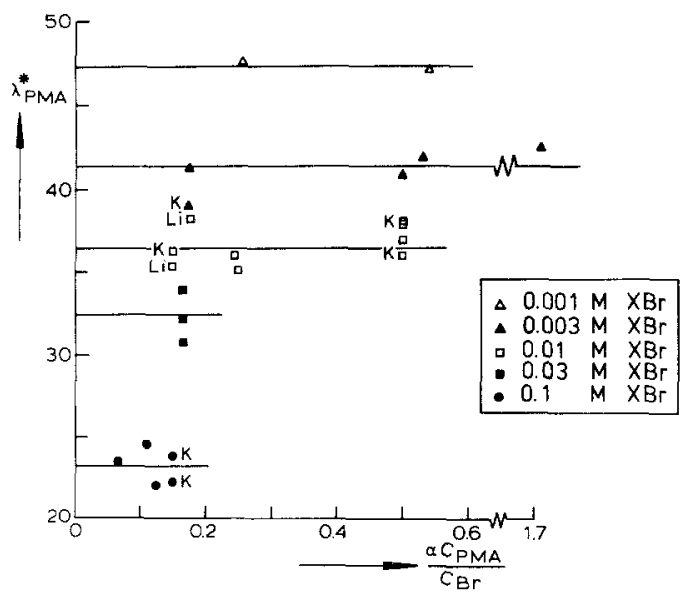

FIG. 3. $\lambda_{\mathrm{PMA}}^{*}$, in $\Omega^{-1} \mathrm{~cm}^{2} \mathrm{eq}^{-1}$, plotted against $\alpha c_{\mathrm{PMA}} / c_{\mathrm{Br}}$ at $\alpha=0.5$. Same symbols as used in Fig. 2.

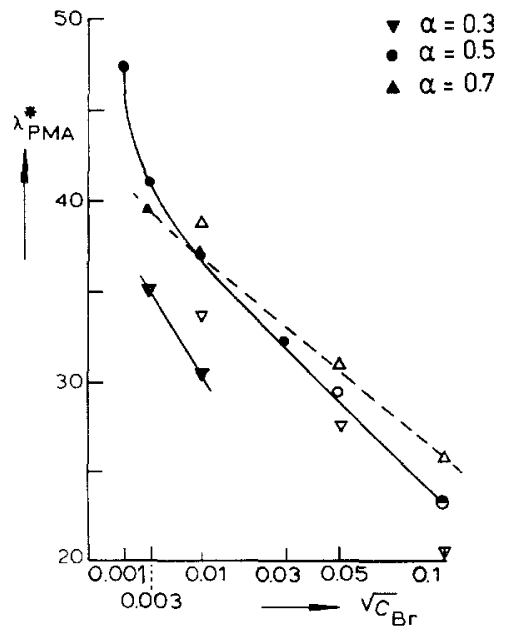

FIG. 4. $\lambda_{\text {PMA }}^{*}$, in $\Omega^{-1} \mathrm{~cm}^{2} \mathrm{eq}^{-1}$, plotted against $c_{\mathrm{Br}}^{1 / 2} \cdot c_{\mathrm{Br}}$ in mole liter $^{-1}$. Open symbols represent data of van Geelen (13).

\section{3b. $\lambda_{P M A}^{*}$}

The equivalent conductivity of the polyion appears not to depend on its concentration in agreement with the theoretical expectations given in the previous paper (4). This is shown in Fig. 3. There is no systematic influence of the choice of the counterion (see Table II). The change of the mobility with the degree of neutralization, $\alpha$, is remarkably small, in agreement with earlier results obtained by van Geelen (13). The slight increase of the mobility with $\alpha$, somewhat steeper below $\alpha=0.5$ than above this value, has also been found for polyacrylic acid $(18,19)$. When all our measurements of $\lambda_{\text {PMA }}^{*}$ at a fixed concentration of bromide and at a fixed degree of neutralization are pooled the results shown in Table II and in Fig. 4 are obtained. It is clear that the PMA mobility decreases strongly with increasing concentration of $\mathrm{XBr}$.

In Table II some results of moving boundary experiments at $5^{\circ} \mathrm{C}$ in $\mathrm{NaCl}$ solutions obtained by van Geelen (13) have been inciuded after a correction for the viscosity difference at the two temperatures (2). The two sets of results are identical within the accuracy of both methods. The 
TABLE II

Equivalent Conductivity, $\lambda \mathrm{NMA}_{\mathrm{A}}^{\mathrm{*}}$, of the Polyions at $25^{\circ} \mathrm{C}^{a}$

\begin{tabular}{|c|c|c|c|c|c|c|c|c|c|c|c|}
\hline \multirow[b]{3}{*}{$\alpha$} & \multicolumn{11}{|c|}{$c(\mathrm{XBr})$} \\
\hline & \multicolumn{2}{|c|}{$0.001 M$} & \multicolumn{2}{|c|}{$0.003 \mathrm{M}$} & \multicolumn{3}{|c|}{$0.01 M$} & \multirow{2}{*}{$\begin{array}{c}0.03 M \\
\mathrm{Na}\end{array}$} & \multirow{2}{*}{$\begin{array}{c}0.05 M \\
\mathrm{Na}\end{array}$} & \multicolumn{2}{|c|}{$0.1 M$} \\
\hline & $\mathrm{Na}$ & $\mathbf{K}$ & $\mathrm{Na}$ & $\mathrm{K}$ & $\mathbf{L i}$ & $\mathrm{Na}$ & $\mathrm{K}$ & & & $\mathrm{Na}$ & $\mathrm{K}$ \\
\hline 0.1 & & & & & & 27.0 & & & 18.4 & 13.9 & \\
\hline 0.2 & & & & & & 28.7 & & & & & \\
\hline \multirow[t]{2}{*}{0.3} & & & 35 & & & 30.6 & 30.2 & & & & \\
\hline & & & & & & 33.8 & & & 26.9 & 20.4 & \\
\hline \multirow[t]{2}{*}{0.5} & 47 & $48^{b}$ & 41.3 & 39 & 36.2 & 36.0 & 36.4 & 32.3 & & 23.4 & 23.0 \\
\hline & & & & & & 37.0 & & & 29.6 & 23.5 & \\
\hline \multirow[t]{2}{*}{0.7} & & & 39.5 & & & 37.0 & 37.2 & & & & \\
\hline & & & & & & 38.8 & & & 30.9 & 25.8 & \\
\hline 0.9 & & & & & & 39.4 & & & 31.6 & 26.9 & \\
\hline
\end{tabular}

${ }^{a}$ The values printed in italics originate from Table 3.1 of van Geelen (13). His measurements were carried out with the moving boundary method at $5^{\circ} \mathrm{C}$ in $\mathrm{NaCl}$ solutions. To make them comparable to our data we multiplied van Geelen's data with the ratio of the viscosities of water $\eta\left(5^{\circ} \mathrm{C}\right) / \eta\left(25^{\circ} \mathrm{C}\right)=1.70 . \lambda_{\mathrm{PMA}}^{*}$ is expressed in $\Omega^{-1} \mathrm{~cm}^{2}$ eq ${ }^{-1}$, where eq means equivalents of neutralized (charged) carboxylic groups.

${ }^{b}$ In $\mathrm{KCl}$ solution (Gelsema, unpublished result of our laboratory).

slightly higher value of van Geelen at $\alpha=0.3$ and at $0.01 M \mathrm{NaCl}$ can be explained since a more compact conformation of PMA is favored at low temperatures and the transition to the more open form occurs just around $\alpha=0.3(20,21)$. Finally, it may not be superfluous to stress that $U_{\mathrm{PMA}}$ $=\lambda_{\mathrm{PMA}} / F$ is the electrophoretic mobility (E.M.) of the polyion.

\section{3c. $\lambda_{\text {counterion }}$ and $\lambda_{\text {counterion }}^{*}$}

The calculation of $\lambda_{\text {counterion }}$ for each individual experiment by subtracting $\lambda_{\mathrm{Br}}$ and $\lambda_{\text {PMA }}$ from the total conductivity, $\kappa$, gives rather inaccurate results, since all errors made in the determination of $\kappa$, $\lambda_{\mathrm{Br}}$, and $\lambda_{\mathrm{PMA}}$ cumulate to a large error in $\lambda_{\text {counterion. }}$. But, if we combine the average value of $\lambda_{\mathrm{PMA}}$ as given in Table II, $\lambda_{\mathrm{Br}}$ as given in Eq. [5], and $\kappa$ as found in a previous paper $(8)$,

$\kappa=\kappa_{\mathrm{XBr}}+\Lambda_{\mathrm{XPMA}}^{0}\left(\alpha c_{\mathrm{XPMA}}\right)$

$$
+B\left(\alpha c_{\mathrm{XPMA}}\right)^{2}
$$

then the equivalent conductivity of the counterion $X$ can be expressed by Eq. [7].

$$
\begin{aligned}
c_{\mathrm{X}} \lambda_{\mathrm{X}}= & \kappa_{\mathrm{XBr}}\left(c_{\mathrm{Br}}, c_{\mathrm{PMA}}=0\right) \\
& +\Lambda_{\mathrm{XPMA}}^{0}\left(\alpha c_{\mathrm{XPMA}}\right)+B\left(\alpha c_{\mathrm{XPMA}}\right)^{2} \\
& -c_{\mathrm{Br}} \lambda_{\mathrm{Br}}\left(c_{\mathrm{Br}}, c_{\mathrm{PMA}}=0\right) \\
& +6\left(\alpha c_{\mathrm{PMA}}\right) \Omega^{-1} \mathrm{~cm}^{2} \text { mole }^{-1} \\
& -\alpha c_{\mathrm{PMA}} \lambda_{\mathrm{PMA}}^{*} \quad \text { (from Table II). [7] }
\end{aligned}
$$

Values for $\Lambda_{\mathrm{XPMA}}^{0}$ and for $B$ can be found in Table I of our paper (8). Most of the counterions $\mathrm{X}$ are introduced into the solution with the supporting electrolyte $\mathrm{XBr}$, but a concentration equivalent to $\alpha c_{\mathrm{PMA}}$ serves to compensate the charge of the polyions. Assuming that the counterions of the supporting electrolyte have their normal mobility, $\lambda_{\mathrm{X}}\left(c_{\mathrm{PMA}}=0\right)$ and that the counterions that neutralize the charge of the polyions and that are localized close to them have a much smaller average mobility, $\lambda_{\mathrm{X}}^{*}$, we split $c_{\mathrm{X}} \lambda_{\mathrm{X}}$ into $c_{\mathrm{Br}} \lambda_{\mathrm{X}}\left(c_{\mathrm{PMA}}=0\right)$ $+\alpha c_{\mathrm{PMA}} \lambda_{\mathrm{X}}^{*}$. We shall consider other choices in the following sections.

Accepting further that

$$
\begin{aligned}
\kappa_{\mathrm{XBr}}\left(c_{\mathrm{Br}}, c_{\mathrm{PMA}}=0\right)= & c_{\mathrm{Br}}\left\{\lambda_{\mathrm{Br}}\left(c_{\mathrm{PMA}}=0\right)\right. \\
& \left.+\lambda_{\mathrm{X}}\left(c_{\mathrm{PMA}}=0\right)\right\},
\end{aligned}
$$


we find Eq. [9] for $\lambda_{\mathrm{X}}^{*}$.

$$
\begin{aligned}
\lambda_{\mathrm{X}}^{*}=\Lambda_{\mathrm{XPMA}}^{0}+6 \Omega^{-1} \mathrm{~cm}^{2} \mathrm{moll}^{-1} & \\
& -\lambda_{\mathrm{PMA}}^{*}+B \alpha c_{\mathrm{PMA}} .
\end{aligned}
$$

For $c_{\mathrm{XBr}}=0.1 \mathrm{M}$, for which the slope of $\lambda_{\mathrm{Br}}$ against $\alpha c_{\mathrm{PMA}} / c_{\mathrm{Br}}$ is rather uncertain, but certainly smaller than $6 \Omega^{-1} \mathrm{~cm}^{2}$ mole ${ }^{-1}$, the term $6 \Omega^{-1} \mathrm{~cm}^{2}$ mole ${ }^{-1}$ in Eq. [9] has to be replaced by a much smaller one or be discarded. Table III gives the values of $\lambda_{\mathrm{x}}^{*}$ extrapolated to zero concentration of PMA.

\section{INTERPRETATION OF THE MOBILITIES OF THE COUNTERIONS, $\lambda_{x}^{*}$}

\section{4a. General Remarks}

When the polymer salt is added to a solution of $\mathrm{XBr}$, the conductivity is changed by a number of effects. The major effects are the contributions of the polymer ions and their counterions to the conductivity, but in addition we have seen that the mobility of the bromide ions originally present is modified and presumably this is also the case for the original $\mathrm{X}$ ions. Concentrating our attention on the change in the conductivity of the supporting electrolyte, we recognize the following four effects.

1. A hydrodynamic effect, due to the entrainment of the ions by the solvent entrained by the polyions, and by backflow compensating this displacement of the polyions. Since the supporting electrolyte is pushed away from the polyions (negative adsorption), the backflow has the more important influence and we should expect a negative contribution, $-h$, to $\lambda_{\mathrm{Br}}$ and an exactly equal positive one, $+h$, to $\lambda_{\mathrm{X}}$. The effect decreases with increasing $c_{\mathrm{XBr}}$ and it has no net influence on the total conductivity.

2. The electric field strength is smaller than average near the polyions, due to the strong relaxation effect, and larger than average in most of the solution. This results in a relative increase by a factor $(1+\Delta)$ of the conductivities of both ions. This effect decreases slightly with increasing $c_{\mathrm{XBr}}$ due to the compression of the ionic atmospheres.

3. There is a presumably small (22) influence of the detour the small ions have to make around the polyion and of the increased friction in their neighborhood, resulting in a relative decrease by a factor $(1-d)$ of the conductivities of both ions.

4. The negative adsorption of the supporting electrolyte leads to an increase in its concentration and thus to an enlarged Debye-Hückel-Onsager effect on the conductivity. By this effect alone the equivalent conductivities of both ions would decrease with amounts

$$
\Delta \lambda_{\mathrm{Br}}=-\left(B_{1} \lambda_{\mathrm{Br}}^{\infty}+B_{2}\right) c_{\mathrm{XBr}}^{1 / 2}\left\{\frac{f \alpha c_{\mathrm{PMA}}}{2 c_{\mathrm{Br}}}\right\}
$$

and

$$
\Delta \lambda_{\mathrm{X}}=-\left(B_{1} \lambda_{\mathrm{X}}^{\infty}+B_{2}\right) c_{\mathrm{X}}^{1 / 2}\left\{\frac{f \alpha c_{\mathrm{PMA}}}{2 c_{\mathrm{Br}}}\right\},
$$

\begin{tabular}{|c|c|c|c|c|c|c|c|c|c|c|c|c|c|c|c|}
\hline \multirow[b]{3}{*}{$\alpha$} & \multicolumn{15}{|c|}{$c(\mathrm{XBr})$} \\
\hline & \multicolumn{3}{|c|}{$0.001 M$} & \multicolumn{3}{|c|}{$0.003 \mathrm{M}$} & \multicolumn{3}{|c|}{$0.01 M$} & \multicolumn{3}{|c|}{$0.03 M$} & \multicolumn{3}{|c|}{$0.1 M$} \\
\hline & $\mathrm{Li}$ & $\mathrm{Na}$ & $\mathrm{K}$ & $\mathrm{Li}$ & $\mathrm{Na}$ & $\mathrm{K}$ & $\mathrm{Li}$ & $\mathrm{Na}$ & $\mathrm{K}$ & $\mathrm{Li}$ & $\mathrm{Na}$ & $\mathrm{K}$ & $\mathrm{Li}$ & $\mathrm{Na}$ & $\mathrm{K}$ \\
\hline 0.3 & - & - & - & - & 15 & 28 & - & 14 & 27 & - & - & - & - & - & - \\
\hline 0.5 & -8 & -2 & +10 & -5 & +2 & +14 & -3 & +3 & +15 & -3 & +5 & +18 & - & +4 & +19 \\
\hline 0.7 & - & - & - & - & -5 & +5 & - & -4 & +6 & - & - & - & - & -1 & +13 \\
\hline
\end{tabular}

TABLE III

Equivalent Conductivity, $\lambda_{\mathrm{x}}^{*}$, of the Counterions that Compensate the Charge of the Polyion ${ }^{a}$

${ }^{a} \lambda_{\mathrm{x}}^{*}$ expressed in $\Omega^{-1} \mathrm{~cm}^{2}$ mole ${ }^{-1}$. 
where $B_{1}$ and $B_{2}$ are the Onsager coefficients and $-\left(B_{1} \lambda^{\infty}+B_{2}\right) C^{1 / 2}$ gives the decrease of the equivalent conductivity between $c=0$ and $c=c . f \alpha c_{\text {PMA }}$ is the negative adsorption of the co-ion, with $f$ a factor between 0.5 and 1.0 .

The four effects together must add up to the term $-\left(6 \alpha c_{\mathrm{PMA}} / c_{\mathrm{Br}}\right) \Omega^{-1} \mathrm{~cm}^{2}$ mole $^{-1}$ in $\lambda_{\mathrm{Br}}$ of Eq. [5] (and to a smaller term at 0.1 $M$ ). At low electrolyte concentrations the backflow effect (1) will be the most important. It will give a negative contribution to $\lambda_{\mathrm{Br}}$, but a positive contribution to $\lambda_{\mathrm{x}}$. As the concentration increases effect (1) will become less important and the negative adsorption (4) will increase in influence, keeping the effect on $\lambda_{\mathrm{Br}}$ about constant, but decreasing the effect on $\lambda_{\mathrm{x}}$. At the highest concentration used by us $(0.1 M)$ the positive effect (2) and the negative effect (4) apparently cancel out for $\mathrm{Br}^{-}$and therefore also combine to only a small effect for $X$.

The above reasoning challenges one of the assumptions made by Möller et al. (5) in the interpretation of polyelectrolyte mobilities and used by us in our previous paper (8) and in our present Eq. [8], namely, that the mobilities of the ions of the supporting electrolyte are unaffected by the introduction of the polyion and its counterions. Unfortunately no quantitative theories are available for effects (1)-(3) and the expressions [10] and [11] for effect (4) are also only first approximations. The best we can do, therefore, is to consider a few extreme limits, leading to modifications of Eq. [9] for $\lambda_{x}^{*}$.

a. Backflow is the overwhelming effect. Then

$$
\lambda_{\mathrm{X}}^{*}=\Lambda_{\mathrm{XPMA}}^{0}-\lambda_{\mathrm{PMA}}^{*}+B\left(\alpha c_{\mathrm{PMA}}\right) .
$$

b. Intermediate case: Effects cancel on $\lambda_{\mathrm{X}}$ (supporting electrolyte $)=\lambda_{\mathrm{X}}\left(c_{\mathrm{PMA}}=0\right)$.

$$
\begin{aligned}
\lambda_{\mathrm{X}}^{*}=\Lambda_{\mathrm{XPMA}}^{0}+6 \Omega^{-1} \mathrm{~cm}^{2} \mathrm{~mole}^{-1} \\
-\lambda_{\mathrm{PMA}}^{*}+B\left(\alpha c_{\mathrm{PMA}}\right) .
\end{aligned}
$$

c. High electrolyte content: field strength effect, (2), important: $\lambda_{\mathrm{Br}}$ (supporting electrolyte $)=\lambda_{\mathrm{Br}}\left(c_{\mathrm{PMA}}=0\right), \lambda_{\mathrm{X}}$ (supporting electrolyte $)-\lambda_{\mathrm{X}}\left(c_{\mathrm{PMA}}=0\right)=$ small $=\delta \lambda\left(\alpha c_{\mathrm{PMA}} / c_{\mathrm{Br}}\right)$, but now $\lambda_{\mathrm{X}}$ depends on $\lambda_{\mathrm{X}}^{\infty}$, i.e., on the choice of the cation.

$$
\begin{aligned}
\lambda_{\mathrm{X}}^{*}=\Lambda_{\mathrm{XPMA}}^{0}-\lambda_{\mathrm{PMA}}^{*} & \\
& +B\left(\alpha c_{\mathrm{PMA}}\right)-\delta \lambda .
\end{aligned}
$$

On any of these three relations the equivalent conductivity of the counterions is low and in several cases even negative. Table III corresponds to Eq. [9]. Equation [12] leads to values which are 6 units lower and Eq. [13] may lead to still slightly lower values, if $\delta \lambda$ is positive.

Rather than interpreting these low mobilities with binding (18) of some of the counterions to the polyion, we prefer an interpretation based on complete dissociation of the COOX groups. In Section 5 we shall see that this assumption allows us to give a quantitative interpretation of the mobility of the PMA ions. The reduced mobility of the $X$ ions is then explained as a consequence of electrophoretic retardation and relaxation effects near the polyions.

\section{4b. Electrophoretic Retardation of the \\ Counterions that Compensate the Charge of the Polyions}

In our paper (8) we derived an average value for the relaxation field strength, $\Delta X$, on and around PMA ions from conductivity data of solutions of $\mathrm{XBr}$ and XPMA. By plotting $\Lambda_{\mathrm{X} \text { PMA }}^{0}$ against $\lambda_{\mathrm{X}}^{\infty}$ at constant concentrations of supporting electrolyte straight lines were obtained with a slope $(1-\Delta X / X)$ (cf. Eqs. [1] and [2] of this paper). It is obvious that exactly the same slopes will be obtained by plotting $\lambda_{\mathrm{x}}^{*}$ either according to Eq. [9] or to Eq. [12] against $\lambda_{\mathrm{X}}^{\infty}$ since (at least for $\alpha c_{\mathrm{PMA}} \rightarrow 0$ ) $\lambda_{\mathrm{X}}^{*}$ and $\Lambda_{\mathrm{XPMA}}^{0}$ differ by a constant amount. Therefore the method of Möller et al. (5) is still applicable for obtaining correct values of $(1-\Delta X / X)$ in the limit of $\alpha c_{\mathrm{PMA}} \rightarrow 0$ even when the mobilities of the supporting electrolyte 
are changed by the presence of PMA. Only when we have to accept Eq. [13] with $\delta \lambda$ being different for different cations, Möller's method leads to slightly incorrect values of $(1-\Delta X / X)$. With the individual mobilities of $\mathrm{X}, \lambda_{\mathrm{X}}^{*}$, available we can go one step beyond the previous results and derive values for the electrophoretic retardation, $\lambda^{\prime}$, of the counterions, using Eq. [1] with the values of $(1-\Delta X / X)$ found previously (8) from conductivities and reproduced in Table $V$ of the present paper. If $\lambda_{x}^{*}$ (for $c_{\mathrm{PMA}} \rightarrow 0$ ) is plotted against $\lambda_{\mathrm{X}}^{\infty}$ for the two or three cations available at constant concentration of the supporting electrolyte the straight lines through the points have slopes $(1-\Delta X / X)$ which agree with the values mentioned above. In Table IV the values of $\lambda_{\mathrm{x}}^{\prime}$ are collected. These electrophoretic retardations are quite large. They increase with increasing $\alpha$ and decrease somewhat with increasing salt concentration. This agrees with the picture that at increasing charge density and increasing salt concentration the counterions come more strongly under the influence of the polyion, but that with increasing salt concentration

\section{TABLE IV}

Electrophoretic Retardation of the Counterions, $\lambda_{\mathrm{x}}^{\prime a}$

\begin{tabular}{rrrrrrrr}
\hline & & \multicolumn{6}{c}{$c(\mathrm{XBr})$} \\
\cline { 3 - 7 }$\alpha$ & $\begin{array}{c}\text { Equa- } \\
\text { tion }\end{array}$ & $0.001 M$ & $0.003 M$ & $0.01 M$ & $0.03 M$ & $0.1 M$ \\
\hline 0.3 & {$[9]$} & - & 22 & 25 & - & - \\
& {$[12]$} & - & 33 & 36 & - & - \\
& & & & & & \\
0.5 & {$[9]$} & 54 & 47 & 45 & 42 & 43 \\
& {$[12]$} & 66 & 59 & 56 & 53 & 53 \\
& & & & & & \\
0.7 & {$[9]$} & - & 61 & 59 & - & 52 \\
& {$[12]$} & - & 75 & 72 & - & 62 \\
\hline
\end{tabular}

\footnotetext{
${ }^{a} \lambda_{\mathrm{X}}^{\prime}$ is expressed in $\Omega^{-1} \mathrm{~cm}^{2}$ mole ${ }^{-1}$. The necessary equivalent conductivities, $\lambda_{X}^{*}$, are calculated, either with Eq. [9] or with Eq. [12]. $\lambda_{\mathrm{X}}\left(c_{\mathrm{Br}} ; c_{\mathrm{PMA}}=0\right)$ and $\lambda_{\mathrm{Br}}\left(c_{\mathrm{Br}} ; c_{\mathrm{PMA}}=0\right)$ are derived from literature data of transport numbers and conductivities (16). The data derived with Eq. [12] are also valid for Eq. [13] with $\delta \lambda=0$.
}

the mobility of the polyions decreases strongly (cf. Fig. 4). Since the electrophoretic retardation is due to entrainment of the counterions by the polyion, it should be smaller than the polyion mobility. A comparison of $\lambda_{\mathrm{X}}^{\prime}$ of Table IV with $\lambda_{\mathrm{PMA}}$ of Table II shows that this is always the case except for $0.1 M \mathrm{XBr}$, if one remembers to multiply $\lambda_{\mathrm{X}}^{\prime}$ with $(1-\Delta X / X)$. In most cases more than half of the polyion velocity is transmitted to the counterions. At $0.1 \mathrm{M}$ $\mathrm{XBr} \lambda_{\mathrm{X}}^{\prime}(1-\Delta X / X)$ is larger than $\lambda_{\mathrm{PMA}}$. This is a first indication that our interpretations have to be modified at $0.1 \mathrm{M}$ and higher concentrations. At the lower concentrations we have some preference for the use of Eq. [9] over that of Eq. [12] mostly on the ground that the somewhat lower electrophoretic retardations seem to be more realistic.

\section{INTERPRETATION OF THE ELECTROPHORETIC MOBILITY OF THE PMA ION}

\section{5a. Dimensions of the Polyion}

From Courtauld's atomic models of isotactic and syndiotactic PMA, we found that isotactic PMA has a nearly circular cross section of $0.7 \mathrm{~nm}$ diameter and a length per monomer of $0.23 \mathrm{~nm}$. Syndiotactic PMA has a cross section with a minimum diameter of $0.6 \mathrm{~nm}$ and a maximum diameter of $0.8 \mathrm{~nm}$. The length per monomer is $0.24 \mathrm{~nm}$. Both models, especially the syndiotactic one, are rather stiff. In our further interpretations we assumed the polyion to be a coiled cylinder with a length per monomer of $0.235 \mathrm{~nm}$ and a radius of $0.35 \mathrm{~nm}(0.3$ and $0.4 \mathrm{~nm}$ are also given for comparison). The actual diameter of the polyion may be larger than the value calculated from the model since, presumably, the $\mathrm{COO}^{-}$groups will be hydrated. The contour length of the molecules of our PMA sample amounts to about $735 \mathrm{~nm}$, more than a thousand times their diameter. End effects are thus reasonably neglected. 


\section{5b. Comparison between Theory and Experiments}

In the preceding paper (4) the electrophoretic mobility (E.M.) of randomly oriented cylinders has been calculated, omitting the relaxation effect. This omission can be corrected for by the method of Möller et al. (5) leading to Eq. [2] for $\lambda$ PMA.

In Table $\mathrm{V}$ we compare calculated values of the E.M., $U$ (calculated), with experimental values, $U^{\text {n.r. }}$, obtained by dividing $\lambda \lambda_{\text {PMA }}^{*}$ as found in Table II by $F(1-\Delta X / X)$ where the relaxation factors $(1-\Delta X / X)$ are taken from our paper (8). The agreement between theory and experiments is quite reasonable for $\alpha=0.5$ and $\alpha=0.7$ and for supporting electrolyte concentrations below $0.1 \mathrm{M}$. The relatively high experimental mobility at $\alpha=0.3$ must be ascribed to the transition of PMA to a more compact form with a lower hydrodynamic resistance. The poor fit at $0.1 M$ bromide will be discussed at the end of the next subsection.

\section{5c. Influence of the Finite Size of the Counterions and of Hydration of the Polyions}

Although the agreement between the experiments and the simple cylinder model with a radius of $0.35 \mathrm{~nm}$ is not bad at all, the model is unrealistic since it neglects the finite size of the counterions and the hydration of the polyion. Because the presence of a Stern layer which takes the finite ion size into account increases the calculated E.M. and hydration of the polyion decreases it, the fit between theory and experiments may be conserved by introducing these two refinements simultaneously.

An estimate of the size of the hydrated alkali ions can be obtained as follows. From a combination of compressibilities and ionic vibration potentials Bockris and Saluja $(23,24)$ have calculated that 4.5

TABLE V

Comparison between Measured Electrophoretic Mobilities, $U^{*}$, and Calculated Values ${ }^{a}$

\begin{tabular}{|c|c|c|c|c|c|c|c|c|c|}
\hline \multirow[b]{2}{*}{$\alpha$} & \multirow{2}{*}{$\begin{array}{c}Q \\
\text { (elem. } \\
\text { charges } \\
\text { per nm) }\end{array}$} & \multirow{2}{*}{$\underset{\text { (mole liter-1) }}{c(\mathrm{XBr})}$} & \multirow{2}{*}{$\begin{array}{c}U^{*} \\
\left(10^{-5} \mathrm{~cm}^{2}\right. \\
\left.\mathrm{V}^{-1} \sec ^{-1}\right)\end{array}$} & \multirow[b]{2}{*}{$1-\Delta X / X$} & \multirow{2}{*}{$\begin{array}{c}U^{\mathrm{n} . r} \\
\left(10^{-5} \mathrm{~cm}^{2}\right. \\
\left.\mathrm{V}^{-1} \sec ^{-1}\right)\end{array}$} & \multicolumn{3}{|c|}{$U$ calculated for } & \multirow{2}{*}{$\underset{(\%)}{\Delta}$} \\
\hline & & & & & & $0.3 \mathrm{~nm}$ & $0.35 \mathrm{~nm}$ & $0.4 \mathrm{~nm}$ & \\
\hline \multirow[t]{3}{*}{0.3} & 1.28 & 0.003 & 36.3 & 0.54 & 67.2 & 64.63 & 61.62 & 59.05 & +9.1 \\
\hline & & 0.01 & 31.5 & 0.56 & 56.3 & 53.10 & 50.25 & 47.81 & +12.0 \\
\hline & & 0.1 & - & 0.54 & - & 33.11 & 30.72 & 28.71 & \\
\hline \multirow[t]{6}{*}{0.5} & 2.13 & 0.001 & 49.2 & 0.52 & 94.7 & 102.40 & 98.62 & 95.36 & -3.8 \\
\hline & & 0.003 & 42.8 & 0.52 & 82.3 & 89.03 & 85.33 & 82.15 & -3.6 \\
\hline & & 0.01 & 37.5 & 0.54 & 69.5 & 74.75 & 71.17 & 68.10 & -2.3 \\
\hline & & 0.03 & 33.5 & 0.57 & 58.7 & 62.19 & 58.76 & 55.84 & -0.1 \\
\hline & & 0.05 & $30.7^{b}$ & $0.59^{c}$ & 52.0 & 56.54 & 53.21 & 50.37 & -2.3 \\
\hline & & 0.1 & 24.0 & 0.63 & 38.1 & 49.14 & 45.96 & 43.26 & -17.1 \\
\hline \multirow[t]{3}{*}{0.7} & 2.98 & 0.003 & 40.9 & 0.42 & 97.4 & 104.04 & 100.13 & 96.76 & -2.7 \\
\hline & & 0.01 & 38.4 & 0.45 & 85.3 & 88.87 & 85.04 & 81.74 & +0.3 \\
\hline & & 0.1 & $26.7^{b}$ & 0.62 & 43.1 & 61.01 & 57.45 & 54.42 & -25.0 \\
\hline
\end{tabular}

${ }^{a}$ By dividing $U^{*}$ by the relaxation factor $(1-\Delta X / X), U^{\text {n.r. }}$ (E.M. with relaxation omitted) is obtained. This value should be compared with the computed value of the E.M. for three different radii of the cylinder $(0.3,0.35$, and $0.4 \mathrm{~nm})$. The last column gives the difference $\Delta$ in percent between $U^{\mathrm{n}, \mathrm{r} \text {. }}$ and the value computed for $0.35 \mathrm{~nm}$ radius.

${ }^{b}$ Mobilities measured by van Geelen and corrected for the temperature difference as described in Table II.

${ }^{c}$ Value interpolated in a plot of $(1-\Delta X / X)$ against $(c(\mathrm{XBr}))^{1 / 2}$. 
water molecules are immobilized around the ions $\mathrm{Li}$ and $\mathrm{Na}$ and 3.8 water molecules around K. A coordination number of 4 water molecules around these alkali ions has been deduced from theoretical calculations (25) in agreement with earlier X-ray observations (26). This suggest that the radius of these hydrated ions can be found by adding the diameter of 1 water molecule $(0.276 \mathrm{~nm})$ to the crystal radii of the ions (Li, $0.060 \mathrm{~nm}$; Na, $0.095 \mathrm{~nm}$; K, $0.133 \mathrm{~nm}$ (27)). This leads to radii of about $0.34 \mathrm{~nm}$ for $\mathrm{Li}, 0.37 \mathrm{~nm}$ for $\mathrm{Na}$, and $0.41 \mathrm{~nm}$ for $\mathrm{K}$. Fortunately, the E.M.'s calculated in this subsection are not critically dependent on the precise values of these radii. Taking $0.375 \mathrm{~nm}$ for the radius of all three ions appears to be quite satisfactory.

Although it is a priori probable that PMA ions are hydrated and that this hydration increases with increasing charge of the polyion we have no independent data on this hydration. We shall assume that the PMA cylinder is surrounded by an immobile shell of water. We shall treat its thickness as an adjustable parameter. The slipping plane is assumed to be situated between this hydration shell and the hydrated counterions, as sketched in Fig. 5. A charge-free Stern layer then stretches from the outer radius of the original PMA to the centers of the first counterions. Calculations of the E.M. as described in our paper (4) then lead to the values of Table VI. The agreement between calculated and measured mobilities is at least as good as that in Table V, if we assume that the thickness of the solvation layer increases from zero at $\alpha=0.3$ via $0.225 \mathrm{~nm}$ at $\alpha=0.5$ to $0.30 \mathrm{~nm}$ at $\alpha=0.7$. We do not assign much significance to the result at $\alpha=0.3$ because of the conformational transition near this value of $\alpha$.

The value found for the thickness is of the order of the diameter of a water molecule, which is reasonable. The ratio (1.44) of the volumes of the hydration layers at $\alpha=0.7$ and $\alpha=0.5$ is very close to the

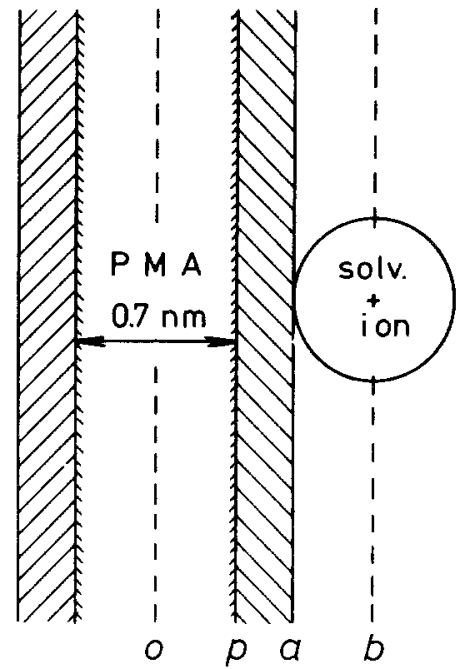

FIG. 5. Section through the axis of a PMA cylinder. $o p=$ radius of PMA $=0.35 \mathrm{~nm} ; p a=$ thickness of hydration layer, varies with $\alpha ; a b=$ radius of hydrated counterions $=0.375 \mathrm{~nm} ; p b=$ charge-free Stern layer; $a=$ slipping plane.

ratio (1.4) of the decrease in volume (electrostriction) accompanying the charging of the PMA chain at these degrees of neutralization (cf. Table 3.V of Ref. (17)).

A variation of the radius of the counterion by $\pm 0.035 \mathrm{~nm}$ leads to an increase or a decrease, respectively, of the calculated E.M. by at most $1.5 \%$ at $0.03 M \mathrm{XBr}$. This small effect justifies the simple assumption of one counterion radius for all three counterions. The fairly large disagreement at $0.1 M \mathrm{XBr}$ is even larger in Table VI than in Table $\mathrm{V}$. We attribute these relatively low E.M.'s to the occurrence of site binding of $20-30 \%$ of the counterions in polyion-water-counterion complexes (28). In order to check this hypothesis we have carried out a few moving boundary experiments using the nonsolvated tetramethylammonium counterion (TMA) in TMABr solutions. The radius of the TMA ion in

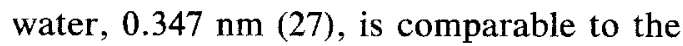
solvated radii of $\mathrm{Li}$ and $\mathrm{Na}$.

We found indeed for $\alpha=0.5$ and $\alpha=0.7$ in $0.1 M \mathrm{TMABr}$ solutions that the E.M. of PMA was about $25 \%$ larger than the 
TABLE VI

Influence of the Finite Size of the Counterions and the Hydration of the Polyion ${ }^{a}$

\begin{tabular}{|c|c|c|c|c|c|c|c|c|}
\hline \multirow[b]{2}{*}{$\alpha$} & \multirow{2}{*}{$\begin{array}{c}Q \\
\text { (elem. } \\
\text { charges } \\
\text { per nm) }\end{array}$} & \multirow{2}{*}{$\begin{array}{c}c(\mathrm{XBr}) \\
\left.\text { (mole liter }{ }^{-1}\right)\end{array}$} & \multirow{2}{*}{$\begin{array}{c}U^{\text {n.r. }} \\
\left(10^{-5} \mathrm{~cm}^{2}\right. \\
\left.V^{-1} \sec ^{-1}\right)\end{array}$} & \multicolumn{4}{|c|}{ Thickness of solvation layer } & \multirow{2}{*}{$\begin{array}{c}\Delta \\
(\%)\end{array}$} \\
\hline & & & & $0 \mathrm{~nm}$ & $0.175 \mathrm{~nm}$ & $0.225 \mathrm{~nm}$ & $0.275 \mathrm{~nm}$ & \\
\hline \multirow[t]{7}{*}{0.5} & 2.13 & 0.001 & 94.7 & 110.33 & 97.63 & 94.89 & 92.41 & -0.2 \\
\hline & & 0.003 & 82.3 & 97.43 & 84.96 & 82.27 & 79.84 & 0 \\
\hline & & 0.01 & 69.5 & 83.91 & 71.73 & 69.12 & 66.80 & +0.5 \\
\hline & & 0.03 & 58.7 & 72.33 & 60.58 & 58.09 & 55.85 & +1.0 \\
\hline & & 0.05 & 52.0 & 67.28 & 55.76 & 53.33 & 51.16 & -2.5 \\
\hline & & 0.1 & 38.1 & 60.87 & 49.77 & 47.45 & 45.39 & -19.7 \\
\hline & & & & $0 \mathrm{~nm}$ & $0.25 \mathrm{~nm}$ & $0.30 \mathrm{~nm}$ & $0.35 \mathrm{~nm}$ & \\
\hline \multirow[t]{3}{*}{0.7} & 2.98 & 0.003 & 97.4 & 122.70 & 101.92 & 99.04 & 96.42 & -1.7 \\
\hline & & 0.01 & 85.3 & 107.99 & 87.54 & 84.72 & 82.72 & +0.7 \\
\hline & & 0.1 & 43.1 & 81.92 & 62.69 & 60.10 & 57.77 & -28.3 \\
\hline
\end{tabular}

${ }^{a} U^{\text {n.r. }}$ as in Table V. Computed values for $0.375 \mathrm{~nm}$ as the radius of the (hydrated) counterions and various thicknesses of the hydration layer around the polyions. The radius of the dry polyion is $0.35 \mathrm{~nm}$. The difference, $\Delta$, in \% between $U^{\text {n.r. }}$ and the calculated value refers to a solvation layer of $0.225 \mathrm{~nm}$ at $\alpha=0.5$ and of $0.30 \mathrm{~nm}$ at $\alpha=0.7$. At $\alpha=0.3$ the best agreement is obtained for zero thickness of the solvation layer, but on account of the conformational transition at this value of $\alpha$ we do not attribute much significance to this result.

values found with the alkali counterions, whereas at $0.03 M \mathrm{TMABr}$ and $\alpha=0.5$ the E.M. was virtually the same as with alkali counterions. Strauss et al. (29) observed analogous phenomena in polyphosphate solutions, where the transition from counterion-independent to counterion-dependent E.M. occurred at $0.08 M$ bromide.

\section{DISCUSSION}

The application of the method of Möller et al. (5) to the estimation of the relaxation effect is based on the supposed additivity of the conductivities of supporting electrolyte and polyelectrolyte. We found, however, that the mobility of the co-ion, bromide, decreased on addition of the polyelectrolyte. In Section $4 \mathrm{a}$ we discussed the implications of this effect on the alkali ion mobility. We could show in Section $4 \mathrm{~b}$ that under the conditions of our experiments, we could still use the above method for finding the relaxation correction $(1-\Delta X / X)$, which was of the order of $50 \%$.
The existence of such strong relaxation effects is supported by conductivity measurements in the megacycle range (30) of a number of flexible polyelectrolytes. These experiments indicate that the relaxation effect is indeed large and of the same order as found by us. The other condition, viz., that the E.M. of PMA is independent of the kind of counterion, was satisfied. The low and in some cases negative mobilities $\left(\lambda_{i}^{*}\right)$ of counterions could be explained by very large electrophoretic retardations, rather than by counterion binding. In solutions of small ions electrophoretic retardation and relaxation effect are calculated separately and linearly superimposed (31), neglecting the cross product between the two effects. In our case the two effects are so large (50\% and more) that it is imperative to take the cross product into account, as we have done in our Eqs. [1] and [2]. We found no need to take counterion binding into account, except at $0.1 \mathrm{M} \mathrm{XBr}$, where site binding was assumed to occur.

The conformational change of PMA at 
about $\alpha=0.3$ made the interpretation of the E.M. at this charge density uncertain. The recent theory by Stigter (7) of the E.M. of cylinders with inclusion of the relaxation effect and its application to our data leads to the conclusion that the observed mobility also at low electrolyte concentration is smaller than that calculated on the assumption of complete contribution of the titrated charge to the electrophoretic charge. Or, in other words, the relaxation effect in Stigter's calculation is smaller than our $\Delta X / X$. Stigter himself mentions some possible reasons for the difference, such as neglect of the relaxation effect of the portions of the coil that are parallel to the applied field and discreteness of the surface charge, and Stigter announces further studies in this area. We prefer to wait for these further developments rather than confront the two approaches in more detail at this moment. We then conclude that conductivity and transference data on alkali PMA in alkali bromide solutions of concentrations up to $0.03 M$ are reasonably interpreted by a model in which the polyion is described as a randomly coiled cylinder, solvated by about a monolayer of water molecules, and in which the counterions carry about four molecules of hydration. The relaxation effect can be approximately found by using the method of Möller et al. (5).

\section{APPENDIX: VISCOSITY MEASUREMENTS}

In order to justify the statement, made in the Introduction, that the interactions between different parts of the coiled cylinder may be neglected, we need an estimate of the diameter of the coil. In their treatment of the viscosity of polymer solutions Debye and Bueche (32) derive a relation between the mean square radius of the porous sphere $\left\langle R_{\text {coil }}^{2}\right\rangle$ that may replace the coil and the mean square radius of gyration $\left\langle s^{2}\right\rangle$.

$$
\left\langle R_{\text {coil }}^{2}\right\rangle=5 / 3\left\langle s^{2}\right\rangle .
$$

TABLE AI

Intrinsic Viscosity, $[\eta]$, of NaPMA at Different Degrees of Neutralization at $25.00^{\circ} \mathrm{C}^{a}$

\begin{tabular}{|c|c|c|c|c|c|c|}
\hline \multirow{2}{*}{$\begin{array}{c}c(\mathrm{NaBr}) \\
(M)\end{array}$} & \multicolumn{2}{|c|}{$\alpha=0.3$} & \multicolumn{2}{|c|}{$\alpha=0.5$} & \multicolumn{2}{|c|}{$\alpha=0.7$} \\
\hline & {$[\eta]$} & $R_{\text {coil }}$ & {$[\eta]$} & $R_{\text {coil }}$ & {$[\eta]$} & $R_{\text {coil }}$ \\
\hline 0.001 & - & - & 1990 & 97 & - & - \\
\hline 0.003 & 1190 & 82 & 1460 & 87.5 & 1460 & 87.5 \\
\hline 0.01 & 641 & 66.5 & 973 & 76.5 & 1000 & 77.0 \\
\hline 0.03 & - & - & 558 & 63.5 & - & - \\
\hline 0.1 & 146 & 40.5 & 310 & 52 & 325 & 53.0 \\
\hline
\end{tabular}

${ }^{a}[\eta]$ is expressed in $\mathrm{cm}^{3} / \mathrm{g}$ polyacid. The radius $R_{\text {coil }} \equiv\left\langle R_{\text {coil }}^{2}\right\rangle^{1 / 2}$ calculated with Eqs. [A1] and [A2] is expressed in $\mathrm{nm}$.

The radius of gyration is related to the intrinsic viscosity $[\eta]$ by

$$
[\eta]=\frac{6^{3 / 2} \Phi\left\langle s^{2}\right\rangle^{3 / 2}}{M},
$$

where $M$ is the molar mass of the polymer and $\Phi$ is a hydrodynamic parameter, the Flory constant (33), whose value depends on the degree of expansion of the coil.

We have used $\Phi=0.86 \times 10^{23} \mathrm{~mole}^{-1}$, the mean value for the system polyacrylate$\mathrm{NaCl}-\mathrm{H}_{2} \mathrm{O}$ (34), also for our system. This value is in good agreement with data of Takahashi et al. (35) for sodium polystyrene sulfonate with $\mathrm{NaCl}$ in water in the corresponding expansion interval. We carried out the necessary viscosity measurements and in order to obtain straight lines in the extrapolation to [ $\eta]$ we used the isoionic dilution technique of Pals and Hermans (36). The necessary parameters for PMA were taken from Strömberg (37). The solutions did not show a deviation from Newtonian behavior.

The intrinsic viscosities and the values for $R_{\text {coil }} \equiv\left\langle R_{\text {coil }}^{2}\right\rangle^{1 / 2}$ calculated with Eqs. [A1] and [A2] are collected in Table AI.

\section{ACKNOWLEDGMENTS}

The authors gratefully acknowledge the contribution of Mr. J. Branger and Mr. H. Bunk in the performance of the Hittorf experiments. They are 
indebted to Miss Paula Both of the NIZO, Ede, The Netherlands, for her assistance in the moving boundary experiments. They thank Mr. A. Reuvecamp for his skillful glassblowing, Mr. W. den Hartog for drawing of the figures, and Miss Henny Miltenburg and Mrs. Marina Uit de Bulten for careful typing of the manuscript.

\section{REFERENCES}

1. Wall, F. T., and Doremus, H., J. Amer. Chem. Soc. 76, 1557 (1954).

2. Eisenberg, H., J. Polym. Sci. 30, 47 (1958).

3. Noda, I., Nagasawa, M., and Ota, M., J. Amer. Chem. Soc. 86, 5075 (1964).

4. van der Drift, W. P. J. T., de Keizer, A., and Overbeek, J. Th. G., J. Colloid Interface Sci. 71, 67-78.

5. Möller, W. J. H. M., van Os, G. A. J., and Overbeek, J. Th. G., Trans. Faraday Soc. 57, 312, 325 (1961).

6. Onsager, L., Physik. Z. 28, 272 (1927).

7. Stigter, D., J. Phys. Chem. 82, 1417, 1424 (1978).

8. van der Drift, W. P. J. T., and Overbeek, J. Th. G., Rec. Trav. Chim. Pays-Bas 98, 81 (1979).

9. Lichtenbelt, J. W. Th., J. Electroanal. Chem. 37, 283 (1972).

10. Brown, A. S., J. Amer. Chem. Soc. 56, 646 (1934).

11. van Laar, J. A. W., thesis, University of Utrecht, The Netherlands, 1952; U. S. A. Patent 2717203.

12. Bijsterbosch, B. H., thesis, University of Utrecht, The Netherlands, 1965.

13. van Geelen, B., thesis, University of Utrecht, The Netherlands, 1958.

14. MacInnes, D. A., and Dole, M., J. Amer. Chem. Soc. 51, 1119 (1929).

15. MacInnes, D. A., and Dole, M., J. Amer. Chem. Soc. 53, 1357 (1931).

16. Landolt-Börnstein, "Zahlenwerte und Funktionen aus Physik, Chemie, Astronomie, Geophysik und Technik," Sechste auflage, II. Band, 7er Teil, Elektrische Eigenschaften II. SpringerVerlag, Berlin, 1960.
17. van der Drift, W. P. J. T., thesis, University of Utrecht, The Netherlands, 1975.

18. Huizinga, J. R., Grieger, P. F., and Wall, F. T., J. Amer. Chem. Soc. 72, 2636 (1950).

19. Nagasawa, M., Noda, I., Takahashi, T., and Shimamoto, N., J. Phys. Chem. 76, 2286 (1972).

20. Leyte, J. C., and Mandel, M., J. Polym. Sci. A2 2, 1879 (1964).

21. Mandel, M., Leyte, J. C., and Stadhouders, M. G., J. Phys. Chem. 71, 603 (1967).

22. Elworthy, P. H., Florence, A. T., and Rahman, A., J. Phys. Chem. 76, 1763 (1972).

23. Bockris, J. O'M., and Saluja, P. P. S., J. Phys. Chem. 76, 2140 (1972).

24. Bockris, J. O'M., and Saluja, P. P. S., J. Phys. Chem. 79, 1230 (1975).

25. Spears, K. G., and Kim, S. H., J. Phys. Chem. 80, 673 (1976).

26. Brady, G. W., J. Chem. Phys. 28, 464 (1958).

27. Robinson, R. A., and Stokes, R. H., "Electrolyte Solutions," Appendix 3.1, Table 6.2. Butterworths, London, 1959.

28. Strauss, U.P., and Leung, Y. P., J. Amer. Chem. Soc. 87, 1476 (1965).

29. Strauss, U. P., Woodside, D., and Wineman, P., J. Phys. Chem. 61, 1353 (1957).

30. Sachs, S. B., Raziel, A., Eisenberg, H., and Katchalsky, A., Trans. Faraday Soc. 65, 77 (1969).

31. Robinson, R. A., and Stokes, R. H., "Electrolyte Solutions," Chap. 7. Butterworths, London, 1959.

32. Debye, P., and Bueche, A. M., J. Chem. Phys. 16, 573 (1948).

33. Flory, P. J., "Statistical Mechanics of Chain Molecules." Wiley-Interscience, New York, 1969.

34. Orofino, T. A., and Flory, P. J., J. Phys. Chem. 63, 283 (1959).

35. Takahashi, A., Kato, T., and Nagasawa, M., J. Phys. Chem. 71, 2001 (1967).

36. Pals, D. T. F., and Hermans, J. J., Rec. Trav. Chim. Pays-Bas 71, 433 (1952).

37. Strömberg, R., Ark. Kemi 25, 117 (1965). 